\title{
Supernova remnants with magnetars: clues to magnetar formation
}

\author{
Jacco Vink \\ Astronomical Institute Utrecht, University Utrecht, P.O. Box 80000, 3508 TA, Utrecht, The Netherlands
}

\begin{abstract}
In this paper I discuss the lack of observational evidence that magnetars are formed as rapidly rotating neutron stars. Supernova remnants containing magnetars do not show the excess of kinetic energy expected for such a formation scenario, nor is there any evidence for a relic pulsar wind nebula. However, it could be that magnetars are formed with somewhat slower rotation periods, or that not all excess rotational energy was used to boost the explosion energy, for example as a result of gravitational radiation. Another observational tests for the rapid initial period hypothesis is to look for statistical evidence that about $1 \%$ of the observed supernovae have an additional $10^{40}-10^{44} \mathrm{erg} / \mathrm{s}$ excess energy during the first year, caused by the spin down luminosity of a magnetar.

An alternative scenario for the high magnetic fields of magnetars is the fossil field hypothesis, in which the magnetic field is inherited from the progenitor star. Direct observational tests for this hypothesis are harder to formulate, unless the neutron star formed in the SN1987A explosion emerges as a slowly rotating magnetar.

Finally, I point out the possible connection between the jets in Cas A and its X-ray point source: the jets in Cas A may indicate that the explosion was accompanied by an X-ray flash, probably powered by a rapidly rotating compact object. However, the point source in Cas A does not seem to be a rapidly rotating neutron star. This suggests that Cas A contains a neutron star that has slowed down considerably in $330 \mathrm{yr}$, requiring a dipole magnetic field of $B>5 \times 10^{13} \mathrm{G}$. The present day lack of evidence for a relic radio pulsar wind nebula may be used to infer an even higher magnetic field of $10^{15} \mathrm{G}$.
\end{abstract}

Key words: stars:neutron and stars:magnetic field and ISM:supernova remnants and ISM:individual:N49 and ISM:individual:Kes 73 and ISM:individual:CTB 109 and ISM:individual:Cas A

\section{Introduction}

Over the last decade "Anomalous X-ray Pulsars" (AXPs) and "Soft Gamma Ray Repeaters" (SGRs) have become one of the most exciting topics in neutron star research (Woods and Thompson, 2004, for a review). The phenomenology of AXPs/SGRs, such as their bursting behavior and their period clustering between $P=5-12 \mathrm{~s}$ have been explained by a variety of mundane and exotic 1 models, including fall-back disks (e.g. Chatteriee and Hernquist, 2000; Alpar, 2001) and the idea that AXPs/SGRs are quarkstars (Ouved et al., 2006; Niebergal et al., 2006). However, the most widely accepted model for AXPs/SGRs is that they are magnetars

Email address: j.vink@astro.uu.nl (Jacco Vink).

1 Both adjectives are not meant to be derogatory; simple, mundane theories are appealing to most scientists, and what is considered exotic changes with time: neutron stars and black holes were once considered very exotic topics.
(Duncan and Thompson, 1992), i.e. neutron stars with ultra high magnetic surface magnetic fields $\left(10^{14}-10^{15} \mathrm{G}\right)$.

The theoretical ideas about magnetars and how they are created were formulated in a series of papers by Duncan and Thompson (Duncan and Thompson, 1992; Thompson and Duncan, 1993; Duncan and Thompson, 1996). They suggested that magnetars form through magnetic field amplification by a powerful dynamo, which was active during the, highly convective, proto-neutron star phase. For this dynamo to operate an initial period is needed that is shorter than the typical convective overturn time of $\sim 3 \mathrm{~ms}$ (Duncan and Thompson, 1996). The time scale in which a magnetar field is formed should be of the order of $10 \mathrm{~s}$.

In this paper I will assume that AXPs/SGRs are indeed magnetars. However, I will discuss whether the most widely accepted theory as to why some neutron stars have very high magnetic fields, namely due to a convective dynamo in a extremely rapidly rotating proto-neutron star, is supported by observational data. Recent observational results have raised some doubt on this canonical magne- 

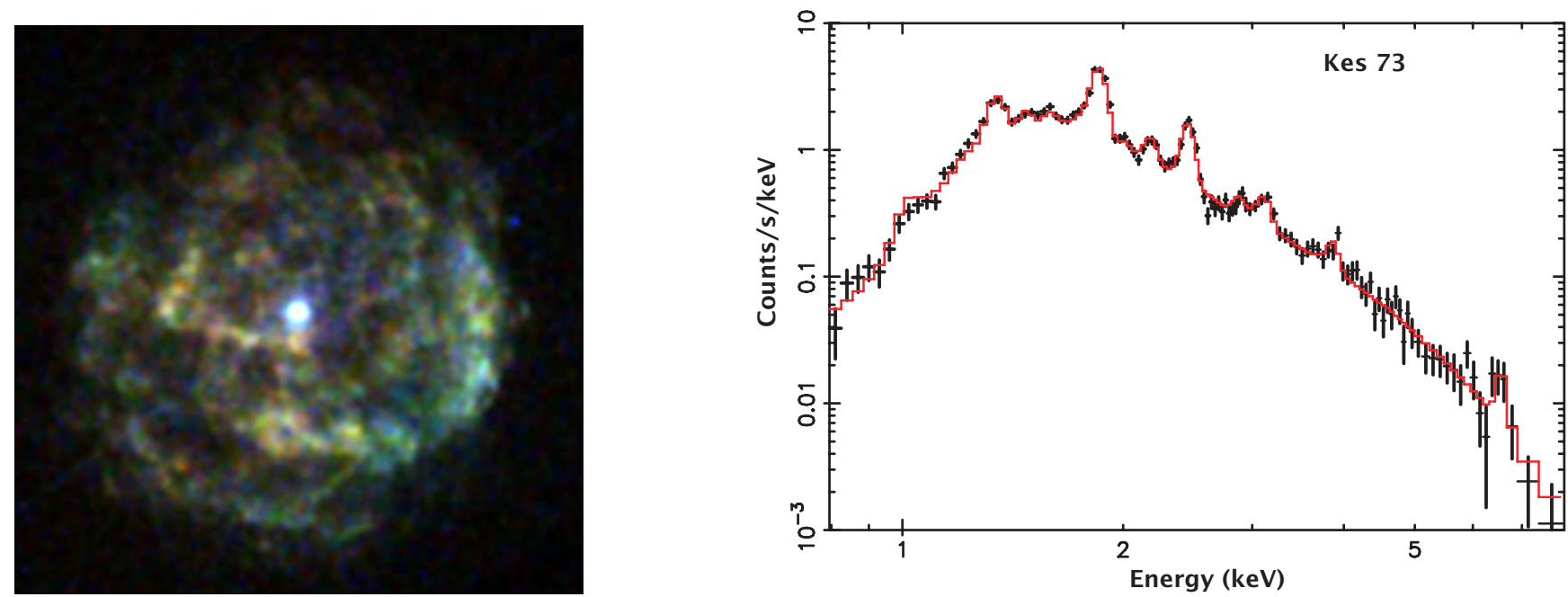

Fig. 1. Left: Chandra X-ray image of Kes 73. The AXP 1E1841-045 is the bright point in the center (saturated in this image in order to bring out the SNR). Right: The XMM-Newton EPIC X-ray spectrum of Kes 73, excluding the AXP. The spectral model consists of a two temperature non-equilibrium ionization model, provided by the SPEX spectral code (Kaastra and Mewe, 2000).

tar formation theory (Ferrario and Wickramasinghe, 2006; Vink and Kuiper, 2006) 2

An alternative theory for magnetar formation is flux conservation, often referred to as the fossil field hypothesis: The high magnetic fields of magnetars directly reflect the high magnetic fields of the cores of their progenitors (Woltier, 1964). In this case magnetar magnetic fields represent the tail of the magnetic field distribution of neutron stars 3

Interestingly, the two hypotheses have opposite implication for the angular momenta of the progenitor stars. The hypothesis of Duncan and Thompson (1992) implies that magnetars are formed from rapidly rotating stellar cores, whereas the fossil field hypothesis implies large stellar magnetic fields, which result in an effective rotational coupling of the stellar core with the stellar surface. In that case stellar winds remove angular momentum from the core, resulting in more slowly rotating stellar cores (Spruit, 2002; Heger et al., 2005).

In that light the debate over magnetic fields and rotation of magnetars is interesting for neutron star initial rotation periods in general. For example the results of Ott et al. (2006) show that short initial pulsar periods are a natural result of core collapse supernovae, only if one ignores the progenitor's magnetic field (see also Heger et al., 2005).

\footnotetext{
2 Note that the quark-star model of Niebergal et al. (2006) also assumes magnetar-like fields for AXPs/SGRs, but assuming initial periods of $5 \mathrm{~s}$. For the fall-back disk model there is no need to invoke high magnetic fields, although hybrid models, a fall-back disk and magnetar-like fields, have also been considered (Ertan et al., 2007). 3 Recently two new theories about magnetar formation have been proposed. Geppert and Rheinhardt (2006) proposed yet another scenario, in which all neutron stars are born with $\sim 10^{15} \mathrm{G}$ fields, but the magnetic field is only stable in those neutron stars that spin faster than $\sim 6 \mathrm{~ms}$ Bhattacharya and Soni (2007) suggests that magnetar fields are created when a phase transition to exotic, magnetized matter occurs in the most massive neutron stars. This model makes the need for a high initial spin period obsolete.
}

Here I will discuss the clues that supernova remnants (SNRs) containing magnetars (AXPs, SGRs) provide us about magnetar formation. This is partially based on the results presented in Vink and Kuiper (2006). However, I will also discuss the X-ray point source in the SNR Cas A, a magnetar candidate. As I will discuss the presence of a putative magnetar in a SNR with a jet/counter jet structure may be an indication that the neutron star was born rapidly rotating.

\section{Supernova remnants and the case for slowly rotating proto-neutron stars}

\subsection{The supernova remnant/magnetar connection}

AXPs and SGRs appear to be very young neutron stars of typically a few thousand years old, as indicated by their spin-down ages. The timing properties of AXPs and SGRs are, however, somewhat erratic, so that characteristic ages are even less reliable age estimators than for other young pulsars. However, the young ages are supported by the fact that of the 12 AXPs and SGRs listed by Woods and Thompson (2004) four are associated with SNRs (see Gaensler, 2004, for a discussion on $\mathrm{SNR} /$ magnetar associations).

The presence of AXPs and SGRs make these SNRs interesting in light of the magnetar formation hypothesis of Duncan and Thompson (1992). As already pointed out by them, and investigated in detail by Thompson et al. (2004) and Bucciantini et al. (2007), a rapidly rotating neutron star may produce very energetic supernovae. The reason is that a large part of the rotational energy of $E_{\text {rot }}=3 \times 10^{52}(P / 1 \mathrm{~ms})^{-2}$ erg will be transfered to the ejecta in less than a few hundred seconds, due to the strong magnetic torque exerted by the neutron star. The association of AXPs/SGRs with SNRs allows us therefore to put 
constraints on the initial rotation period, by investigating whether these SNRs are more energetic than other SNRs (c.f. Allen and Horvath, 2004; Arons, 2003).

\subsection{Supernova remnant energies and magnetar initial spin periods}

Vink and Kuiper (2006) investigated whether SNRs containing AXPs/SGRs are more energetic by compiling and/or determining the energies of the SNRs Kes 73 (AXP 1E 1841-045), CTB 109 (AXP 1E2259+586) and N49 (SGR 0526-66). Not investigated was G29.6+0.1, a very faint SNR associated with candidate AXP AX J1845-045 (Vasisht et al., 2000). The quality of the archival X-ray data of this SNR is unfortunately too poor for spectroscopic analysis.

The energies of SNRs can be estimated from their Xray spectra, by determining the plasma temperature (an indication for the shock velocity), emission measure (an indication for the density) in conjunction with an estimate of the distance. The results are summarized Table 1.

It is clear that the energies of these three SNRs are all consistent with the canonical supernova energy of $10^{51} \mathrm{erg}$ (but see section 2.3). The method for estimating explosion energies is well tested, see for example Hughes et al. (1998), who found explosion energies for other SNRs that are comparable to the energies listed in Table 1 . The method assumes that the SNRs are in the Sedov phase of their evolution, which strictly speaking only applies to older SNRs. However, for the young SNR Kes 73 Vink and Kuiper (2006) also considered the Truelove and McKee (1999) model for young SNRs, which confirm the relatively low energy of Kes 73.

The implication is that the birth of magnetars cannot have resulted in an additional energy input of more than $10^{51} \mathrm{erg}$, which corresponds to $P_{i} \gtrsim 5 \mathrm{~ms}$. This is therefore evidence against the hypothesis that magnetars are formed with initial periods of $P_{i} \lesssim 3 \mathrm{~ms}$. However, this conclusion is only valid if most of the rotational energy ended up as kinetic energy of the ejecta. Moreover, it may also indicate that the convective dynamo is still effective for initial periods slightly longer than $3 \mathrm{~ms}$.

\subsection{The distance to CTB 109}

[ It must be pointed out that the estimated explosion energies scale with the distance, as $d^{2.5}$. Very recently, Durant and van Kerkwiik (2006) showed that the distance to CTB 109 may be twice as large, placing AXP $1 \mathrm{E} 2259+586$ in the Outer Spiral Arm $(d \sim 7.5 \mathrm{kpc})$ instead of in the Perseus Arm $(d \sim 3 \mathrm{kpc})$, and suggesting an explosion energy of $7 \times 10^{51} \mathrm{erg}$ for CTB 109. The distance measurement consists of comparing the optical absorption of nearby field stars with the X-ray absorption of the AXP. For this the correlation between $N_{\mathrm{H}}$ versus $A_{V}$ is used of Predehl and Schmitt (1995).
Although the results of Durant and van Kerkwijk (2006) are interesting, the new distance measurement should be used cautiously. First of all, there is an intrinsic spread in the $N_{\mathrm{H}}-A_{V}$ relation. Secondly, Durant and van Kerkwijk (2006) use the $N_{\mathrm{H}}$ measured from the AXP, which is much higher than that derived for the SNR $\left(1.1 \times 10^{22} \mathrm{~cm}^{-2}\right.$ versus $\left.0.7 \times 10^{22} \mathrm{~cm}^{-2}\right)$. Adopting the latter would result in a distance consistent with the previously derived distance of $\sim 3 \mathrm{kpc}$ (Kothes et al., 2002).

The likely reason for the discrepancy between the AXP's and SNR's absorption is a large variation of absorption columns in this region. Indeed, CTB 109 seems to be interacting with a molecular cloud (Sasaki et al., 2004) and it may be that this molecular cloud is partly in front of the AXP. The nearby SNR Cas A has a similarly high variation in absorption. Interestingly, Cas A is only 2 degrees away from CTB 109 and closer to the Galactic plane. Its distance is $3.4 \mathrm{kpc}$ (Reed et al., 1995), which places it at the far-side of the Perseus Arm. However, its absorption column is larger than that of $1 \mathrm{E} 2259+586$, namely $N_{H}=$ $1.3 \times 10^{22} \mathrm{~cm}^{-2}$. This makes the new distance estimate for CTB 109 all the more peculiar.

The case for a larger distance to CTB 109 is, in my view, not yet compelling, but it is important to investigate the distance with different methods: An energy of $10^{51} \mathrm{erg}$ suggests $P_{i} \lesssim 5 \mathrm{~ms}$, whereas $7 \times 10^{51} \mathrm{erg}$ is consistent with $P_{i}<3 \mathrm{~ms}$ the limit at which significant magnetic field amplification in the proto-neutron star can take place (Duncan and Thompson, 1996).

\section{Rotational energy losses}

Using the SNR energy to constrain the initial spin period of magnetars rests on the assumption that a large fraction of the rotational energy will eventually be dumped into the supernova ejecta (e.g. Thompson et al., 2004). It may be possible that instead rotational energy escapes in the form of a jet, due to losses in the form of gravitational radiation, or due to perhaps another yet unknown mechanism.

However, any loss mechanism needs to operate in a time that is shorter than the magnetic braking time scale, but comparable to, or longer than the time scale for magnetic field amplification, in order not to interfere with the magnetic amplification process: According to Duncan and Thompson (1992) the emerging magnetic field damps out differential rotation in less than $10 \mathrm{~s}$. In vacuum, the magnetic braking time scale is $\sim 4 \times 10^{3}(P / 1 \mathrm{~ms})^{-2}\left(B_{p} / 10^{15} \mathrm{G}\right)^{-2}$ s. However, Thompson et al. (2004) have shown that for realistic conditions the time scale for rotational energy loss may be shorter than $<100 \mathrm{~s}$, and if a relativistic wind emanates from the proto-neutron star it may even be $<30 \mathrm{~s}$.

So whatever loss mechanism removes rotational energy without in the end converting it into kinetic energy, it has to operate on a time scale between $10 \mathrm{~s}$ and less than several hundred seconds. 
Table 1

The explosion energies and ages of the supernova remnants from X-ray spectral analysis (after Vink and Kuiper (2006), the pulsar dipole field has been taken from Woods and Thompson (2004)).

\begin{tabular}{|c|c|c|c|c|c|c|c|c|c|}
\hline SNR/Pulsar & $\begin{array}{c}\text { Distance } \\
\mathrm{kpc}\end{array}$ & $\begin{array}{l}\text { radius } \\
\text { pc }\end{array}$ & $\begin{array}{c}\mathrm{E} \\
\left(10^{51} \mathrm{erg}\right)\end{array}$ & $\begin{array}{c}n_{\mathrm{H}} \\
\mathrm{cm}^{-3}\end{array}$ & $\begin{array}{c}\text { Mass } \\
M_{\odot}\end{array}$ & $\begin{array}{c}\text { SNR Age } \\
10^{3} \mathrm{yr}\end{array}$ & $\begin{array}{c}\text { Pulsar Ageb } \\
10^{3} \mathrm{yr}\end{array}$ & $\begin{array}{c}B_{d} \\
10^{14} \mathrm{G}\end{array}$ & References \\
\hline Kes 73/1E1841-045 & 7.0 & 4.3 & $0.5 \pm 0.3$ & $2.9 \pm 0.4$ & $29 \pm 4$ & $1.3 \pm 0.2$ & 4.3 & 7.1 & Vink and Kuiper (2006) \\
\hline CTB109/1E2259+586 & 3.0 & 10 & $0.7 \pm 0.3$ & $0.16 \pm 0.02$ & $97 \pm 23$ & $8.8 \pm 0.9$ & 220 & 0.6 & Sasaki et al. (2004) \\
\hline N49/SGR 0526-66 & 50 & 9.3 & $1.3 \pm 0.3$ & $2.8 \pm 0.1$ & $320 \pm 50$ & $6.3 \pm 1.0$ & 1.9 & 7.4 & Vink and Kuiper (2006) \\
\hline
\end{tabular}

Distances and pulsar ages $\left(\tau=\frac{1}{2} P / \dot{P}\right)$ are taken from Woods and Thompson (2004).
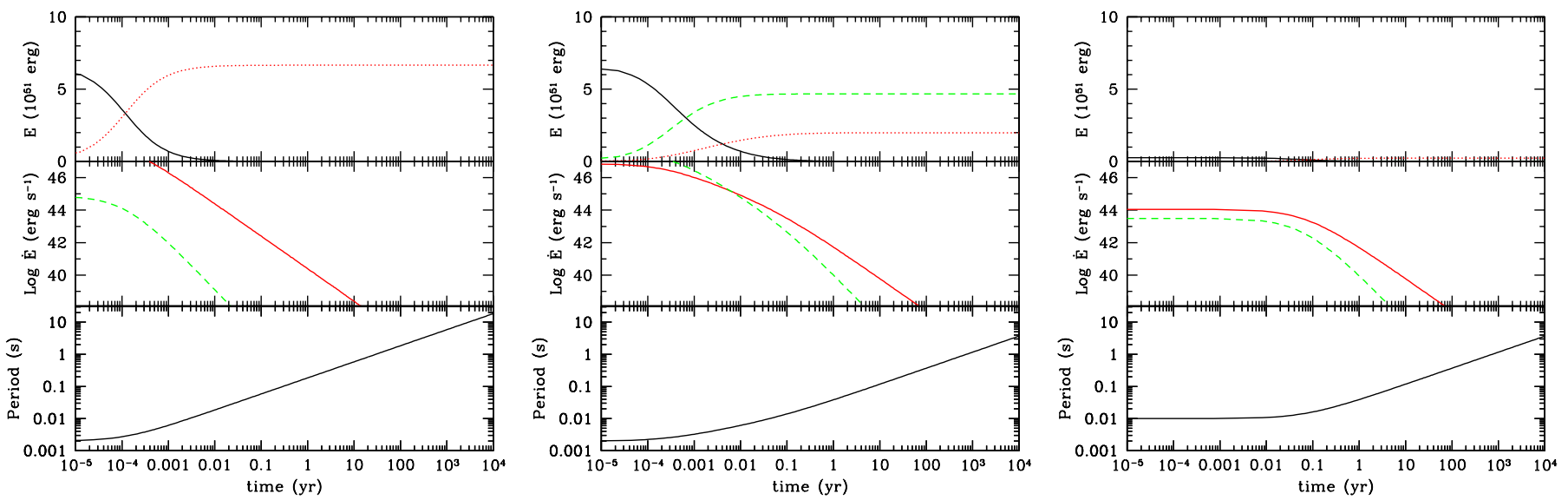

Fig. 2. Simple spindown models for magnetars, assuming the spindown scenario of Stella et al. (2005) with different values for the interior and dipole magnetic field. From left to right: a) $\left.B_{\text {int }}=10^{16} \mathrm{G} / B_{d}=10^{15} \mathrm{G}, P_{0}=2 \mathrm{~ms} ; \mathrm{b}\right) B_{\text {int }}=5 \times 10^{16} \mathrm{G} / B_{d}=2 \times 10^{14} \mathrm{G}$,

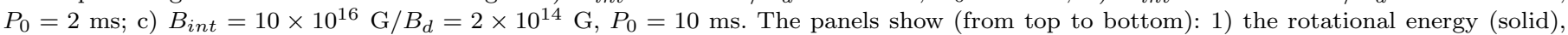

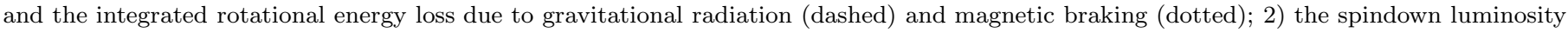
in gravitational radiation, and dipole radiation (for young radio pulsars resulting in a relativistic wind of similar magnitude); 3 ) the pulse period history. For comparison a supernova has a peak luminosity of $10^{42}-10^{43} \mathrm{erg} \mathrm{s}^{-1}$.

\subsection{Magnetars and Jets}

Jets are a common phenomenon in almost every accretion process in astrophysics, from young stellar objects to active galactic nuclei. It is therefore quite natural to assume that jets may also form during the core-collapse process. It has even been argued that jets may be a key ingredient for the supernova explosion itself (e.g. Akivama et al., 2003 ). However, even if a jet forms it will in most cases not survive the passage through the star. Exceptions are, of course, supernovae associated with gamma-ray bursts and $\mathrm{X}$-ray flashes. In fact, it has been argued that the X-ray flash XRF 060218 was driven by the birth of a magnetar (Mazzali et al., 2006).

So could it be that magnetar creation is accompanied by jet formation, which takes away most of the rotational energy, rather than that the rotational energy drives the ejecta: 4

Indeed, it is possible that X-ray flashes are the result of jet formation associated with magnetar formation, as I will discuss in section 5. However, there is no evidence that the

\footnotetext{
4 Jet formation may seem a natural outcome of accretion processes, but jets are unlikely to provide an efficient mechanism for angular momentum transport, if aligned with the angular momentum vector.
}

birth of magnetars, in general, results in the formation of powerful jets: The morphology of the SNRs listed in Table 1 do not show any evidence for jets. Kes 73 does not even show signs of asphericity (Fig. 1).

One may of course wonder whether the explosion was jet induced, but that by now the shock has become more spherical. However, this would bring us back to question why SNRs with magnetars have not more energetic SNR shells.

It is intriguing, though, that the SNR Cas A shows ample evidence for a bipolar jet structure (see section 5), and it has been argued that the mysterious point source in Cas $\mathrm{A}$ is a magnetar: its X-ray emission properties and lack of detectable radio emission is reminiscent of AXPs/SGRs (Chakrabarty et al., 2001), and an infrared light echo of a putative giant SGR flare has been detected (Krause et al., 2005).

\subsection{Gravitational waves}

One way for a rapidly spinning neutron star to lose its rotational energy without powering the supernova ejecta is through gravitational radiation. Stella et al. (2005) recently showed that magnetar formation may be accompa- 
nied by gravitational radiation, provided that the internal magnetic field is unaligned with the rotation axis, $B_{i} \gtrsim$ $5 \times 10^{16}$, and the dipole magnetic field is relatively low, $B_{d}<5 \times 10^{14} \mathrm{G}$. The reason is that gravitational radiation is caused by a deformation of neutron star caused by interior magnetic stresses. The dipole magnetic field at the surface determines the magnitude of the competing process, i.e. magnetic braking. Note, however, that Stella et al. (2005) assume magnetic braking in vacuum, which is far from realistic (Thompson et al., 2004).

Moreover, the dipole surface magnetic field needs to be small; smaller than the observed magnetic fields of some AXPS/SGRs. This could mean either that the magnetic field is initially highly disordered, and dominated by higher order multipoles, or the magnetic field is buried for some time (e.g. Geppert et al., 1999). However, if field emergence is slow, it will be harder to explain the slow rotational periods of AXPs and SGRs.

The most direct observational test for the idea that magnetar birth is accompanied by gravitational radiation is to detect gravitational waves, coinciding with a supernova. The rotational frequency and its decay with $\dot{\omega} \propto \omega^{5}$ should be a clear signature of such an event. As shown by Stella et al. (2005), the likelihood for detecting such an event in the Galaxy with future gravitational wave detectors is small, but the signal may be strong enough to detect supernovae as far out as the Virgo cluster.

For the moment we have no means of directly testing whether magnetars are born with $P<3 \mathrm{~ms}$ periods and subsequently lose much of this energy in the form of gravitational waves. However, even though gravitational radiation losses may be dominant in the first few minutes, the "rapidly spinning proto-neutron star" magnetar formation scenario may still have other observational consequences.

\subsection{Further observational tests for rapid initial rotation}

Since rotational energy loss by gravitational radiation depends more strongly on the angular momentum than magnetic braking, sooner or later magnetic braking $(\dot{\omega} \propto$ $\omega^{3}$ ) will dominate over gravitational wave losses. Using the equations used by Stella et al. (2005), and assuming an internal field of $5 \times 10^{16} \mathrm{G}$ and a dipole field of $10^{14} \mathrm{G}$, one finds that by the time the period is $12 \mathrm{~ms}$ magnetic braking will be the most important mechanism for angular momentum loss. Because all pulsars with high spin down luminosities produce pulsar wind nebula $(\mathrm{PWN})$, one may wonder why we do not detect PWNe around magnetars.

The situation of young radio pulsars and magnetars is not quite comparable: magnetic braking in magnetars is much more rapid. So the Crab nebula has taken $1000 \mathrm{yr}$ to make the powerful synchrotron nebula, but a rapidly rotating magnetar will form a relativistic wind bubble within a year, that is, during the supernova rather than the SNR phase. In the first few days, instead of creating a PWN, magnetic braking will probably directly propel the ejecta (the scenario considered by Vink and Kuiper, 2006), but as soon as the ejecta have expanded sufficiently, a relativistic wind will create a bubble of relativistic particles within the supernova, similar to a PWN. Depending on the density of the ejecta this PWN may directly heat the ejecta, causing a prolonged brightness of the supernova, or it may create a high luminosity PWN, inside the ejecta. Again depending on the density of the ejecta, the UV/X-ray synchrotron radiation may be reprocessed and result in bright optical/infrared supernovae, or it will create a very bright radio and X-ray source. The low energy part of the relativistic electron population, which do not suffer as much synchrotron losses, may survive for a long time, and may still be visible as a fossil radio PWN. The presence of the radio PWN may depend on the strength of the magnetar dipole field in its first ten years. For very strong fields $\left(\sim 10^{15} \mathrm{G}\right)$ the pulsar slows down to $P=0.5 \mathrm{~s}$ during the supernova phase, in which the freely expanding ejecta are still dense and may influence the shape and evolution of the (radio) PWN. For $B_{d} \sim 10^{14} \mathrm{G}$ a PWN is still formed after the supernova phase has seized and a more normal PWN may be formed, whose radio relic should still be observable.

The enhanced brightness of supernovae is only mildly dependent on the emission of strong gravitational radiation, or the exact initial spin period, as long as $P_{i} \lesssim 10 \mathrm{~ms}$. For strong magnetic dipole fields, the supernova's luminosity may not have an enhanced plateau, but will instead be brighter in the first months. One can judge this from the various spin down scenarios in Fig. 2, which shows, for relatively modest dipole fields, a spin down luminosity of $10^{42}-10^{40} \mathrm{erg} \mathrm{s}^{-s}$ in the first 1 to $10 \mathrm{yrs}$.

The picture sketched above needs to be modeled more rigorously, which is well worth the effort, since it may lead to two observational tests for magnetar formation scenarios, even for $3 \mathrm{~ms} \lesssim P_{i} \lesssim 10 \mathrm{~ms}: 1$ ) are there supernovae that are exceptionally bright for more than a year, either in Xrays or in the optical? 2) are young magnetars surrounded by fossil radio PWN?

None of the known magnetars seem to be surrounded by relic radio PWNe. The AXP 1E1841-045 lies even within a local minimum of the radio emission of the SNR Kes 73 (Kriss et al., 1985).

As far as I know, there is no evidence for supernovae that are unusually bright for about a year, except perhaps for SN 1988A in M58, which showed according to Ruiz-Lapuente et al. (1991) a departure from the normal radio-active decay powered light curve. However, this was disputed by Turatto et al. (1993). Investigating this issue in a more systematic way would require long term (1-2 year) monitoring of nearby supernovae in the optical and $\mathrm{X}$-rays. In essence it provides a statistical test: given that there are about 220 Galactic core collapse SNRs and only 3 are associated with magnetars one expects that about $1 \%$ of the core collapse supernovae should show the effects of a rapidly rotating magnetar.

Finally, it may be worthwhile to investigate whether radio emission from supernovae (Weiler et al., 2005) may in 
some cases be the result of a forming and expanding PWN, rather than the result of an interaction of the supernova shock with the progenitor wind. From this point of view, the radio brightening of SN1987J (Bietenholz and Bartel, 2007) is interesting as it may tell us about the initial spin period of a putative pulsar formed by the supernova.

\section{The fossil field hypothesis}

Given the lack of evidence for the rapid spinning scenario for magnetar formation so far, it is interesting to look into the alternative possibility: the fossil field hypothesis, i.e. the magnetar field is a result of the high magnetic field in the core of the progenitor star. The fossil field hypothesis can be divided in two hypotheses: 1) the strong fossil field hypothesis, which assumes that the strong progenitors magnetic field is a result of the strong magnetic field of the cloud from which the star formed (Ferrario and Wickramasinghe, 2005, 2006), and 2) the progenitors magnetic field is a result of magnetic field amplification inside the progenitor.

From an observational point of view the (weak and strong) fossil field hypothesis is well worth considering. It is well known that about $5 \%$ of the A stars have a high magnetic field, 1-1000 kG, constituting the Ap class. Type A stars are progenitors of white dwarfs and from a statistical point of view it seems plausible that Ap stars are the progenitors of magnetic white dwarfs (Ferrario and Wickramasinghe, 2005). This also indicates that stellar magnetic fields are stable enough to survive during the life time of the star.

Observationally it is much harder to determine magnetic fields of $\mathrm{O}$ and $\mathrm{B}$ stars, the progenitors for neutron stars. However, for a few $\mathrm{O}$ and $\mathrm{B}$ stars high magnetic fields have been reported. Interestingly,the B0.2V star $\tau$ Sco (Donati et al., 2006b) and the O star HD 191612 (Donati et al., 2006a), both magnetic stars, seem to have long rotation periods of 41 and 538 days, respectively. According to Donati et al. (2006b), this argues in favor of the fossil field hypothesis for reasons already mentioned in the introduction: if the magnetic field is due to a dynamo process, one needs convective motions in conjunction with a short rotation period.

There are, however, still many unsolved questions regarding magnetic fields in massive stars. First of all, for the fossil field theory it is unclear how a magnetic field can survive the turbulent formation process of the star. On the other hand, even the average magnetic field in the interstellar medium must have an influence on the star forming process, in the sense that strict flux conservation would result in magnetic fields that provide pressure support against collapse (Mestel and Spitzer, 1956). Forming stars therefore need to dissipate or expulse magnetic field energy. A one to one relation between local interstellar magnetic field and stellar magnetic field seems therefore rather naive.

Another issue is the stability of the magnetic field. Even if high magnetic fields are observed in O stars, will this magnetic field survive during the life time of the star? This may depend on the magnetic field topology; apparently, some magnetic field configurations, a mix of dipolar and toroidal components, are stable against fast decay (Braithwaite and Spruit, 2004). Concerning the inevitable slow decay, one may speculate that since more massive stars live shorter, they can end their life with a higher magnetic field. Within the framework of the fossil field origin for neutron magnetic fields this may explain observational evidence that magnetars form from the most massive stars (Gaensler et al., 2005).

Finally, all measurements of magnetic field in stars concerns the surface magnetic fields. However, the neutron star magnetic field, according to the fossil field theory comes from the stellar core. The evidence for a connection between magnetic white dwarfs and Ap stars gives some reassurance that indeed high magnetic stars have high magnetic cores, but the evidence is of a statistical nature. For magnetars the fossil field hypothesis is also hard to prove directly. One potential piece of evidence would, however, be if a very young magnetar would have already a long period. One hypothetical case would be if the neutron star formed in SN1987A turns out to be a slowly rotating neutron star. However, no neutron star has yet been detected in SN1987A, but interestingly, there is also no evidence yet of a powerful pulsar (Haberl et al., 2006). The current upper limits on the X-ray luminosity from a putative pulsar are consistent with a bipolar magnetic field of $10^{14} \mathrm{G}$ and an initial period close to $0.5\left(B / 10^{14}\right)^{1 / 2} \mathrm{~s}$. This should be considered as an alternative hypothesis next to a more rapidly spinning pulsar with a low magnetic field, as discussed by Haberl et al. (2006).

\section{Was the point source in Cas $\mathrm{A}$ a rapidly spinning proto-neutron star?}

There are a number of SNRs that contain unresolved Xray sources, which are likely to be neutron stars with some unusual properties: they show no evidence for radio emission, and are not surrounded by PWNe. They therefore resemble AXPs, but since no pulsation period has been detected they cannot be positively identified as magnetars. Moreover, their surface temperatures seem lower than those of AXPs (Pavlov et al., 2004). We are hiding our ignorance about these sources by assigning them to a new class: central compact objects (CCOs). The fact that CCO's are not surrounded by PWN suggests that their rotational energy loss is low, meaning that either they have a very low surface magnetic field, or, perhaps more likely, they are slow rotators.

The canonical CCO is the X-ray point source in Cas A, detected in the first light image of Chandra (Tananbaum, 1999). Recently, the Spitzer infrared observatory found that infrared emission from the vicinity of the SNR shows evidence for (super)luminal motion (Krause et al., 2005), attributed to a light echo caused by a luminous outburst of 
the CCO in the 1950's. This would imply that the point source is indeed a magnetar.

What makes the point source in Cas A equally fascinating is that it may be a magnetar in a SNR, which also shows the presence of jets (Vink, 2004; Hwang et al., 2004; Hines et al., 2004; Fesen et al., 2006). The jets have an energy of $\sim 10^{50} \mathrm{erg}$ (Laming et al., 2006, see also Schure et al. 2007 , in preparation), whereas the total supernova explosion energy was probably $2 \times 10^{51} \mathrm{erg}$ (Laming and Hwang, 2003). The explosion energy, the ejecta mass $\left(2-4 M_{\odot}\right.$, Vink et al., 1996; Willingale et al., 2002) and the jet energies are remarkably similar to the properties derived for the X-ray flash associated with SN 2006aj (Mazzali et al., 2006).

Usually it is assumed that gamma-ray bursts are formed from the collapse of a rapidly rotating stellar core into a black hole (collapsar theory MacFadyen et al., 2001), whereas their weaker counterparts, the X-ray flashes, may be similar, but instead a rapidly spinning neutron star is formed. So how does this relate to the fact that the point source in Cas A is likely to be a slowly rotating neutron star? The answer may be that the neutron star has slowed down considerably in the 330 yrs of its existence, as a result of magnetic braking.

If the jets obtained their energy of $\sim 10^{50} \mathrm{erg}$ from the rotation of the neutron star, as argued by Wheeler et al. (2002), this would imply an initial period of at least $17 \mathrm{~ms}$. In order to calculate a lower limit to the bipolar magnetic field we need to estimate from the non-detection of a PWN a lower limit to the current pulsar period. We can do this as follows. The rotational energy of a neutron star is $E_{\text {rot }}=\frac{1}{2} I \Omega^{2}$, with $I=1.4 \times 10^{45} \mathrm{~g} \mathrm{~cm}^{2}$, thus

$\dot{E}_{r o t}=I \Omega \dot{\Omega}=2 E_{\text {rot }} \frac{\dot{P}}{P}=\frac{E_{r o t}}{\tau}$,

with $\tau=P /(2 \dot{P})$, the characteristic pulsar age $($ Seward and
$1988)$. For Cas A we can set $\tau=330 \mathrm{yr}$, thereby assuming that the neutron star was born with $P_{i} \ll P(t=330 \mathrm{yr})$. Most of the visible energy losses of the PWN will occur in X-rays, with typically $L_{X} \approx \dot{E} / 100$. Despite deep X-ray images of Cas A (e.g. Hwang et al., 2004) no PWN has been detected, so we may safely say that the X-ray continuum luminosity in the 4-6 continuum band is from the remnant shell, as shown by the X-ray images (e.g. Vink and Laming, 2003). The $\mathrm{X}$-ray continuum above $1 \mathrm{keV}$ is approximately a power law up to energies of $\sim 80 \mathrm{keV}$ with $L_{X}(1-80 \mathrm{keV})=4 \times 10^{36} \mathrm{erg} \mathrm{s}^{-1}$ (e.g. Vink and Laming, 2003; Renaud et al., 2006). Consequently, the PWN must have a total luminosity substantially less than that. If we conservatively say that the PWN has $L_{X}<10^{36} \mathrm{erg} \mathrm{s}^{-1}$ ( $25 \%$ of the overall X-ray continuum), we obtain according to Eq. $1 \dot{E}<1 \times 10^{38} \mathrm{erg} \mathrm{s}^{-1}$, and $E_{\text {rot }}<1.0 \times 10^{48} \mathrm{erg}$, corresponding to a current pulsar period of $P>160 \mathrm{~ms} 5$

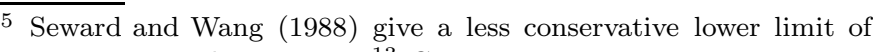
$P>330 \mathrm{~ms}$, and $B>7 \times 10^{13} \mathrm{G}$.
For a neutron star to slow down from a period of $\sim 17 \mathrm{~ms}$ to a period of $>160 \mathrm{~ms}$ in $330 \mathrm{yr}$ a bipolar magnetic field is necessary of $>5 \times 10^{13} \mathrm{G}$, suggesting magnetic fields of magnetar strength, or a bit less. This extremely interesting given the recent suggestions that X-ray flashes are powered by newly born magnetars (Mazzali et al., 2006; Soderberg et al., 2006). One problem, has to be solved in that case: if the pulsar once had a much higher rotational energy loss, why don't we observe a fossil radio PWN? As discussed in section 3.3 the answer may be that the magnetic field of the magnetar is $\sim 10^{15} \mathrm{G}$, the PWN will then form inside the dense, freely expanding ejecta during the first ten years of its life.

\section{Summary and conclusions}

Magnetars are one of the hottest topics (literally!) in neutron star research. It is usually assumed that they are formed from rapidly rotating proto-neutron stars, and that their magnetic field is the result of a dynamo acting in the first few seconds of the neutron stars life (Duncan and Thompson, 1992).

However, as I have discussed, there is not yet any observational evidence for this: the SNRs containing magnetars are not more energetic than other SNRs, excluding a very rapid initial rotation ( $P \gtrsim 5 \mathrm{~ms}$, Vink and Kuiper, 2006). Moreover, the fossil field theory offers a compelling alternative, since we know that magnetic $\mathrm{O}$ and $\mathrm{B}$ stars exist, and it is also likely, given the plausible connection between magnetic A stars and magnetic white dwarfs.

Nevertheless, at this point nothing can be stated with any certainty about magnetar magnetic field creation. It could be that magnetar fields are formed by a convective dynamo, even if initial rotations are slightly longer than $3 \mathrm{~ms}$, or part of the rotational energy is lost in the form of angavitational radiation.

However, I have pointed that the possibilities for testing magnetar formation scenarios has not yet been exhausted. One might look for the energy input from a rapidly rotating magnetar in supernovae, which should occur in about $1 \%$ of the observed core collapse supernovae, or one might look for (faint) relic radio PWN surrounding the known magnetars.

The fossil field hypothesis is more difficult to directly test, but one possibility would be to find a slowly rotating magnetar in a very young SNR. A possibility is that the neutron star formed during the SN1987A explosion may turn out to be a slowly rotating magnetar $(P \gtrsim 0.5 \mathrm{~s})$.

Finally, I have pointed out that Cas A may be an X-ray flash remnant. Since jet formation likely requires a rapidly rotating compact object (Wheeler et al., 2002), the X-ray point source in Cas A may once have been a rapid rotator $(P \lesssim 17 \mathrm{~ms})$. The present day lack of a detectable X-ray pulsar wind nebula, implying $P>160 \mathrm{~ms}$, would therefore require it to be a magnetar-like magnetic field $B>5 \times$ $10^{13} \mathrm{G}$. In order to avoid the creation of a relic radio PWN an even higher (initial) magnetic field needs to be inferred 
of $B \sim 10^{15} \mathrm{G}$.

\section{References}

Akiyama, S., Wheeler, J. C., Meier, D. L., Lichtenstadt, I., Feb. 2003. The Magnetorotational Instability in CoreCollapse Supernova Explosions. ApJ 584, 954-970.

Allen, M. P., Horvath, J. E., Nov. 2004. Influence of an Internal Magnetar on Supernova Remnant Expansion. ApJ 616, 346-356.

Alpar, M. A., Jun. 2001. On Young Neutron Stars as Propellers and Accretors with Conventional Magnetic Fields. ApJ 554, 1245-1254.

Arons, J., Jun. 2003. Magnetars in the Metagalaxy: An Origin for Ultra-High-Energy Cosmic Rays in the Nearby Universe. ApJ 589, 871-892.

Bhattacharya, D., Soni, V., May 2007. A Natural Explanation for Magnetars. ArXiv e-prints 705.

Bietenholz, M., Bartel, N., 2007. The Evolution of the Central Component in SN1986J. AdSpR (this volume).

Braithwaite, J., Spruit, H. C., Oct. 2004. A fossil origin for the magnetic field in A stars and white dwarfs. Nat 431, 819-821.

Bucciantini, N., Quataert, E., Arons, J., Metzger, B. D., Thompson, T. A., May 2007. Magnetar Driven Bubbles and the Origin of Collimated Outflows in Gamma-ray Bursts. ArXiv e-prints 705.

Chakrabarty, D., Pivovaroff, M. J., Hernquist, L. E., Heyl, J. S., Narayan, R., Feb. 2001. The Central X-Ray Point Source in Cassiopeia A. ApJ 548, 800-810.

Chatterjee, P., Hernquist, L., Nov. 2000. The Spin Period, Luminosity, and Age Distributions of Anomalous X-Ray Pulsars. ApJ 543, 368-372.

Donati, J.-F., et al., Jan. 2006a. Discovery of a strong magnetic field on the O star HD 191612: new clues to the future of $\theta^{1}$ Orionis $\mathrm{C}^{*}$. MNRAS 365, L6-L10.

Donati, J.-F., et al., Aug. 2006b. The surprising magnetic topology of $\tau$ Sco: fossil remnant or dynamo output? MNRAS 370, 629-644.

Duncan, R. C., Thompson, C., Jun. 1992. Formation of very strongly magnetized neutron stars - Implications for gamma-ray bursts. ApJ 392, L9-L13.

Duncan, R. C., Thompson, C., 1996. Magnetars. In: Rothschild, R. E., Lingenfelter, R. E. (Eds.), AIP Conf. Proc. 366: High Velocity Neutron Stars. pp. 111-+.

Durant, M., van Kerkwijk, M. H., Jun. 2006. Distances to Anomalous X-ray Pulsars using Red Clump Stars. ArXiv Astrophysics e-prints.

Ertan, Ü., Erkut, M. H., Ekşi, K. Y., Alpar, M. A., Mar. 2007. The Anomalous X-Ray Pulsar 4U 0142+61: A Neutron Star with a Gaseous Fallback Disk. ApJ 657, 441447.

Ferrario, L., Wickramasinghe, D., Mar. 2006. Modelling of isolated radio pulsars and magnetars on the fossil field hypothesis. MNRAS , 292-+.
Ferrario, L., Wickramasinghe, D. T., Jan. 2005. Magnetic fields and rotation in white dwarfs and neutron stars. MNRAS 356, 615-620.

Fesen, R. A., et al., Jul. 2006. The Expansion Asymmetry and Age of the Cassiopeia A Supernova Remnant. ApJ 645, 283-292.

Gaensler, B. M., 2004. Anomalous X-ray pulsars and soft gamma-ray repeaters - the connection with supernova remnants. Advances in Space Research 33, 645-653.

Gaensler, B. M., McClure-Griffiths, N. M., Oey, M. S., Haverkorn, M., Dickey, J. M., Green, A. J., Feb. 2005. A Stellar Wind Bubble Coincident with the Anomalous XRay Pulsar 1E 1048.1-5937: Are Magnetars Formed from Massive Progenitors? ApJ 620, L95-L98.

Geppert, U., Page, D., Zannias, T., May 1999. Submergence and re-diffusion of the neutron star magnetic field after the supernova. A\&A 345, 847-854.

Geppert, U., Rheinhardt, M., Sep. 2006. Magnetars versus radio pulsars. MHD stability in newborn highly magnetized neutron stars. A\&A 456, 639-649.

Haberl, F., Geppert, U., Aschenbach, B., Hasinger, G., Dec. 2006. XMM-Newton observations of ¡ASTROBJ ¿SN $_{\text {S }}$ $1987 \mathrm{~A}_{\mathrm{i}} /$ ASTROBJ $_{i}$. A\&A 460, 811-819.

Heger, A., Woosley, S. E., Spruit, H. C., Jun. 2005. Presupernova Evolution of Differentially Rotating Massive Stars Including Magnetic Fields. ApJ 626, 350-363.

Hines, D. C., et al., Sep. 2004. Imaging of the Supernova Remnant Cassiopeia A with the Multiband Imaging Photometer for Spitzer (MIPS). ApJS 154, 290-295.

Hughes, J. P., Hayashi, I., Koyama, K., Oct. 1998. ASCA X-Ray Spectroscopy of Large Magellanic Cloud Supernova Remnants and the Metal Abundances of the Large Magellanic Cloud. ApJ 505, 732-748.

Hwang, U., et al., Nov. 2004. A Million Second Chandra View of Cassiopeia A. ApJ 615, L117-L120.

Kaastra, J. S., Mewe, R., Oct. 2000. Coronal Plasmas Modeling and the MEKAL code. In: Bautista, M. A., Kallman, T. R., Pradhan, A. K. (Eds.), Atomic Data Needs for X-ray Astronomy. p. 161.

Kothes, R., Uyaniker, B., Yar, A., Sep. 2002. The Distance to Supernova Remnant CTB 109 Deduced from Its Environment. ApJ 576, 169-175.

Krause, O., et al., Jun. 2005. Infrared Echoes near the Supernova Remnant Cassiopeia A. Science 308, 1604-1606.

Kriss, G. A., Becker, R. H., Helfand, D. J., Canizares, C. R., Jan. 1985. G27.4+0.0 - A galactic supernova remnant with a central compact source. ApJ 288, 703-706.

Laming, J. M., Hwang, U., Nov. 2003. On the Determination of Ejecta Structure and Explosion Asymmetry from the X-ray Knots of Cassiopeia A. ApJ 597, 347-361.

Laming, J. M., Hwang, U., Radics, B., Lekli, G., Takács, E., Jun. 2006. The Polar Regions of Cassiopeia A: The Aftermath of a Gamma-Ray Burst? ApJ 644, 260-273.

MacFadyen, A. I., Woosley, S. E., Heger, A., Mar. 2001. Supernovae, Jets, and Collapsars. ApJ 550, 410-425.

Mazzali, P. A., et al., Aug. 2006. A neutron-star-driven Xray flash associated with supernova SN 2006aj. Nat 442, 
1018-1020.

Mestel, L., Spitzer, Jr., L., 1956. Star formation in magnetic dust clouds. MNRAS 116, 503.

Niebergal, B., Ouyed, R., Leahy, D., Jul. 2006. Magnetic Field Decay and Period Evolution of Anomalous X-Ray Pulsarsin the Context of Quark Stars. ApJ 646, L17-L20.

Ott, C. D., Burrows, A., Thompson, T. A., Livne, E., Walder, R., May 2006. The Spin Periods and Rotational Profiles of Neutron Stars at Birth. ApJS 164, 130-155.

Ouyed, R., Niebergal, B., Dobler, W., Leahy, D., Dec. 2006. Three-Dimensional Simulations of the Reorganization of a Quark Star's Magnetic Field as Induced by the Meissner Effect. ApJ 653, 558-567.

Pavlov, G. G., Sanwal, D., Teter, M. A., 2004. Central Compact Objects in Supernova Remnants. In: IAU Symposium. pp. 239.

Predehl, P., Schmitt, J. H. M. M., Jan. 1995. X-raying the interstellar medium: ROSAT observations of dust scattering halos. A\&A 293, 889-905.

Reed, J. E., Hester, J. J., Fabian, A. C., Winkler, P. F., Feb. 1995. The three-dimensional structure of the Cassiopeia A supernova remnant. I. The spherical shell. ApJ 440, 706-+.

Renaud, M., et al., Aug. 2006. The Signature of ${ }^{44} \mathrm{Ti}$ in Cassiopeia A Revealed by IBIS/ISGRI on INTEGRAL. ApJ 647, L41-L44.

Ruiz-Lapuente, P., Kidger, M., Gomez, G., Canal, R., Lopez, R., Sep. 1991. SN 1988A in M58 - Departure from Co-56 decay 700 days after explosion. ApJ 378, L41-L44.

Sasaki, M., Plucinsky, P. P., Gaetz, T. J., Smith, R. K., Edgar, R. J., Slane, P. O., Dec. 2004. XMM-Newton Observations of the Galactic Supernova Remnant CTB 109 (G109.1-1.0). ApJ 617, 322-338.

Seward, F. D., Wang, Z.-R., Sep. 1988. Pulsars, X-ray synchrotron nebulae, and guest stars. ApJ 332, 199-205.

Soderberg, A. M., et al., Aug. 2006. Relativistic ejecta from X-ray flash XRF 060218 and the rate of cosmic explosions. Nat 442, 1014-1017.

Spruit, H. C., Jan. 2002. Dynamo action by differential rotation in a stably stratified stellar interior. A\&A 381, 923-932.

Stella, L., Dall'Osso, S., Israel, G. L., Vecchio, A., Dec. 2005. Gravitational Radiation from Newborn Magnetars in the Virgo Cluster. ApJ 634, L165-L168.

Tananbaum, H., Sep. 1999. Cassiopeia A. IAU circ. 7246, 1.

Thompson, C., Duncan, R. C., May 1993. Neutron star dynamos and the origins of pulsar magnetism. ApJ 408, 194-217.

Thompson, T. A., Chang, P., Quataert, E., Aug. 2004. Magnetar Spin-Down, Hyperenergetic Supernovae, and Gamma-Ray Bursts. ApJ 611, 380-393.

Truelove, J. K., McKee, C. F., Feb. 1999. Evolution of Nonradiative Supernova Remnants. ApJS 120, 299-326.

Turatto, M., Cappellaro, E., Benetti, S., Danziger, I. J., Nov. 1993. Observations of Type-II Plateau Supernovae - Supernova 1988A Supernova 1988H and Supernova 1989C. MNRAS 265, 471-+.
Vasisht, G., Gotthelf, E. V., Torii, K., Gaensler, B. M., Oct. 2000. Detection of a Compact X-Ray Source in the Supernova Remnant G29.6+0.1: A Variable Anomalous X-Ray Pulsar? ApJ 542, L49-L52.

Vink, J., Feb. 2004. X- and $\gamma$-ray studies of Cas A: exposing core collapse to the core. New Astronomy Review 48, 6167.

Vink, J., Kaastra, J. S., Bleeker, J. A. M., Mar. 1996. A new mass estimate and puzzling abundances of SNR Cassiopeia A. A\&A 307, L41-L44.

Vink, J., Kuiper, L., Jul. 2006. Supernova remnant energetics and magnetars: no evidence in favour of millisecond proto-neutron stars. MNRAS 370, L14-L18.

Vink, J., Laming, J. M., Feb. 2003. On the magnetic fields and particle acceleration in Cassiopeia A. ApJ 584, 758 769.

Weiler, K. W., van Dyk, S. D., Sramek, R. A., Panagia, N., Stockdale, C. J., Montes, M. J., Dec. 2005. Radio Emission from Supernovae. In: Turatto, M., Benetti, S., Zampieri, L., Shea, W. (Eds.), ASP Conf. Ser. 342: 16042004: Supernovae as Cosmological Lighthouses. pp. 290+ .

Wheeler, J. C., et al., Apr. 2002. Asymmetric Supernovae from Magnetocentrifugal Jets. ApJ 568, 807-819.

Willingale, R., Bleeker, J. A. M., van der Heyden, K. J., Kaastra, J. S., Vink, J., Jan. 2002. X-ray spectral imaging and Doppler mapping of Cassiopeia A. A\&A 381, 1039 1048.

Woltjer, L., Oct. 1964. X-Rays and Type I Supernova Remnants. ApJ 140, 1309-1313.

Woods, P., Thompson, C., 2004. Soft gamma repeaters and anomalous X-ray pulsars: Magnetar candidates. in Compact Stellar X-ray Sources, eds. W.H.G. Lewin and M. van der Klis (astro-ph/0406133). 